\title{
Analysis on Nitrogen and Phosphorus Removal of Sand Stone Dam in River
}

\author{
Hong-Cui WANG ${ }^{1, a,{ }^{*}}$, Dao-Tan WANG ${ }^{1, b}$, Zhu BAO ${ }^{1, \mathrm{c}}$ and Xu-Shen ZHOU ${ }^{1, \mathrm{~d}}$ \\ Haihe River Water Conservancy Commission, Tianjin, 300170, China \\ alalycui1981@126.com, bwangdaotan@126.com, cbaozhu@hwcc.gov.cn, \\ zhouxushen@hwcc.gov.cn \\ ${ }^{*}$ Corresponding author
}

Keywords: Sand and stone filter dam, Water quality, Nitrogen and phosphorus removal.

\begin{abstract}
The water quality of Panjiakou Reservoir is greatly affected by the upstream water quality. A large number of waste water containing nitrogen and phosphorus enter the Panjiakou Reservoir through the main stream of Luanhe River, resulting in the increasing eutrophication. Four sand and stone filter dams were constructed in the main stream of Luanhe River. Through the analysis of continuous monitoring data, the river was purified through four dams and the effect of nitrogen and phosphorus removal was remarkable. The mechanism and service life of nitrogen and phosphorus removal of sand and stone filter dam need to be further studied.
\end{abstract}

\section{Introduction}

With the rapid development of industrial and agricultural production, after rainwater washing and infiltration, a large number of waste water containing nitrogen and phosphorus nutrients entered rivers, lakes and reservoirs, resulting in nitrogen and phosphorus outstanding, and eutrophication are becoming more and more serious.

The water quality of Panjiakou Reservoir is greatly affected by the upstream water quality. On the one hand, there are three districts and eight counties of Chengde City, which in the upstream of Panjiakou Reservoir. A large number of untreated industrial wastewater and urban domestic sewage are discharged into the main stream of the Luan River and then into the Panjiakou Reservoir. On the other hand, a large number of pesticides and fertilizers enter the Panjiakou Reservoir through the upstream river with the torrential rain runoff [1]. The eutrophication degree of the Panjiakou Reservoir is becoming more and more serious; because of the upstream river entering the Panjiakou Reservoir, which is rich in nitrogen and phosphorus nutrients. Therefore, it is very important to research on nitrogen and phosphorus removal by sand and stone filter dam for improve the water quality and slow down the eutrophication of Panjiakou reservoir through in Luan River.

\section{Description of the Study Area}

The Panjiakou Reservoir is located in northern Hebei Province on the Luan River, supplies drinking water to the city of Tianjin. The Luan River has its origins near the border between Hebei Province and Inner Mongolia. The total area of the Luan River watershed is $54,530 \mathrm{~km}^{2}$. The mean annual runoff volume is 2.45 billion $\mathrm{m}^{3}$ of water. Construction of the reservoir began in 1975 and storage of water in 1979. Hydroelectric power generation began in 1984. The length of the Panjiakou dam is $1039 \mathrm{~m}$ and has a maximum height of $107.5 \mathrm{~m}$. The total storage capacity of Panjiakou Reservoir is 2.93 billion $\mathrm{m}^{3}$. The total surface area of the reservoir, if full, would be $70 \mathrm{~km}^{2}$, but the usual areal extent is $40 \mathrm{~km}^{2}$.

Agriculture might have a significant impact on the quality of reservoir water because of the large amounts of fertilizes used. Because of the amount of land in corn production, the input of nitrogen and phosphorus is of concern, especially during the summer monsoon season. Industrial uses of water may also affect water quality in Panjiakou Reservoir. The industries that produce sewage in Chengde 
include food, papermaking, chemical fertilizer, brewage, metallurgy, dye printing, mineral dressing, and production of pharmaceuticals.

\section{Sand and Stone Filter Dam}

\section{Design}

In order to improve the water quality of Panjiakou Reservoir, four sand and stone filter dams were built on Luan River, about $30 \mathrm{~km}$, between the Chengqiangyu and the Mangyushao in the Xinglong County of Chengde City in the upper reaches of the Panjiakou Reservoir, which mainly deal with below $15 \mathrm{~m}^{3} / \mathrm{s}$ discharge.

After stone-throwing in the river course, dry block stone filter dam is filled with sand gravel and small diameter stone. The size of sand and stone filter dam 1 is $80 \mathrm{~m}$ long, $1.2 \mathrm{~m}$ high and $8.0 \mathrm{~m}$ wide, the dam 2 is $65 \mathrm{~m}$ long, $1.5 \mathrm{~m}$ high and $8.0 \mathrm{~m}$ wide, the dam 3 is $50 \mathrm{~m}$ long, $2.0 \mathrm{~m}$ high and $8.4 \mathrm{~m}$ wide, the dam 4 is $60 \mathrm{~m}$ long, $1.6 \mathrm{~m}$ high and $8.0 \mathrm{~m}$ wide. The detailed construction drawings are shown in figure 1-4, and the dimensions in the drawings are all in $\mathrm{mm}$.

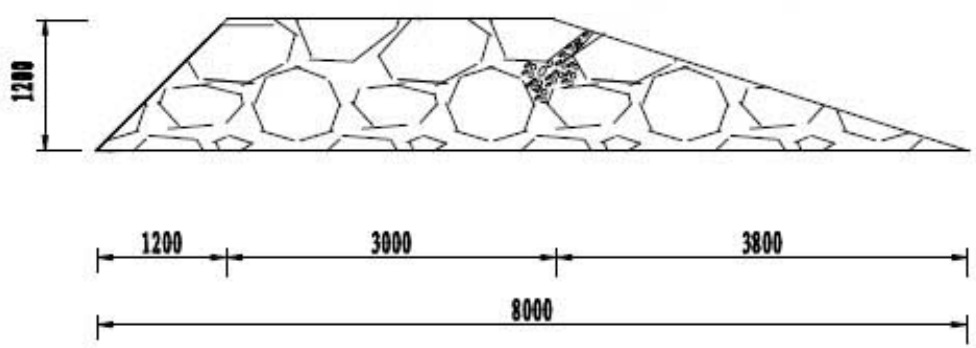

Fig. 1 Sand and stone filter dam 1 construction drawing (length of dam $80 \mathrm{~m}$ )

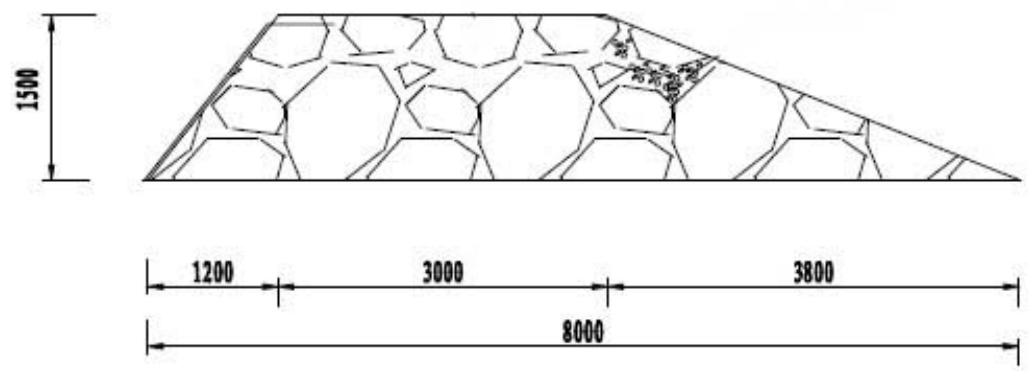

Fig. 2 Sand and stone filter dam 2 construction drawing (length of dam 65 m)

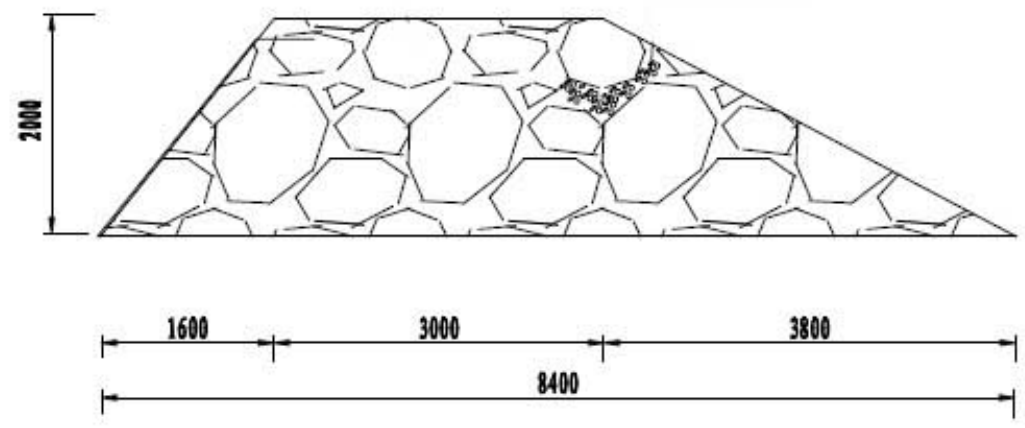

Fig. 3 Sand and stone filter dam 3 construction drawing (length of dam $50 \mathrm{~m}$ ) 


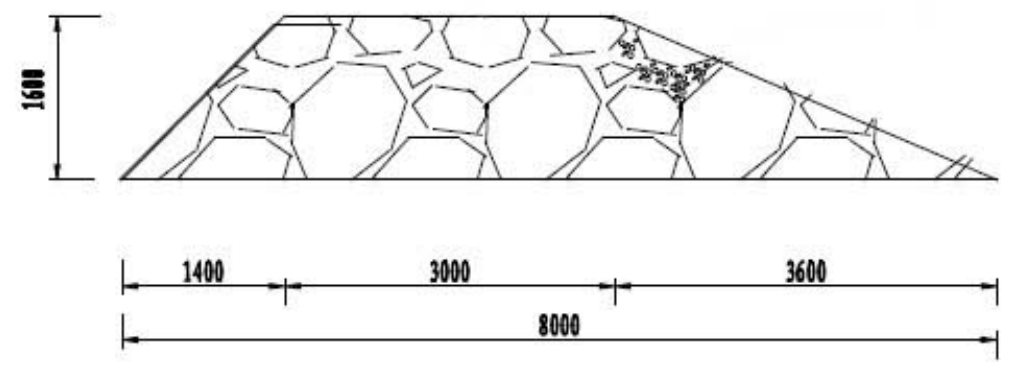

Fig. 4 Sand and stone filter dam 4 construction drawing (length of dam $60 \mathrm{~m}$ )

\section{Construction}

The total length of the river is $0.5 \mathrm{~m}$ deep and $25 \mathrm{~m}$ wide and $15 \mathrm{~m}$ long, with a total excavation capacity of $1000 \mathrm{~m}^{3}$. Then about $2000 \mathrm{~m}^{3}$ of stone, cobblestone, and coarse sand fillers are filled into four river sections respectively, and the 4 sand and stone filter dams formed are shown in figure 5 .
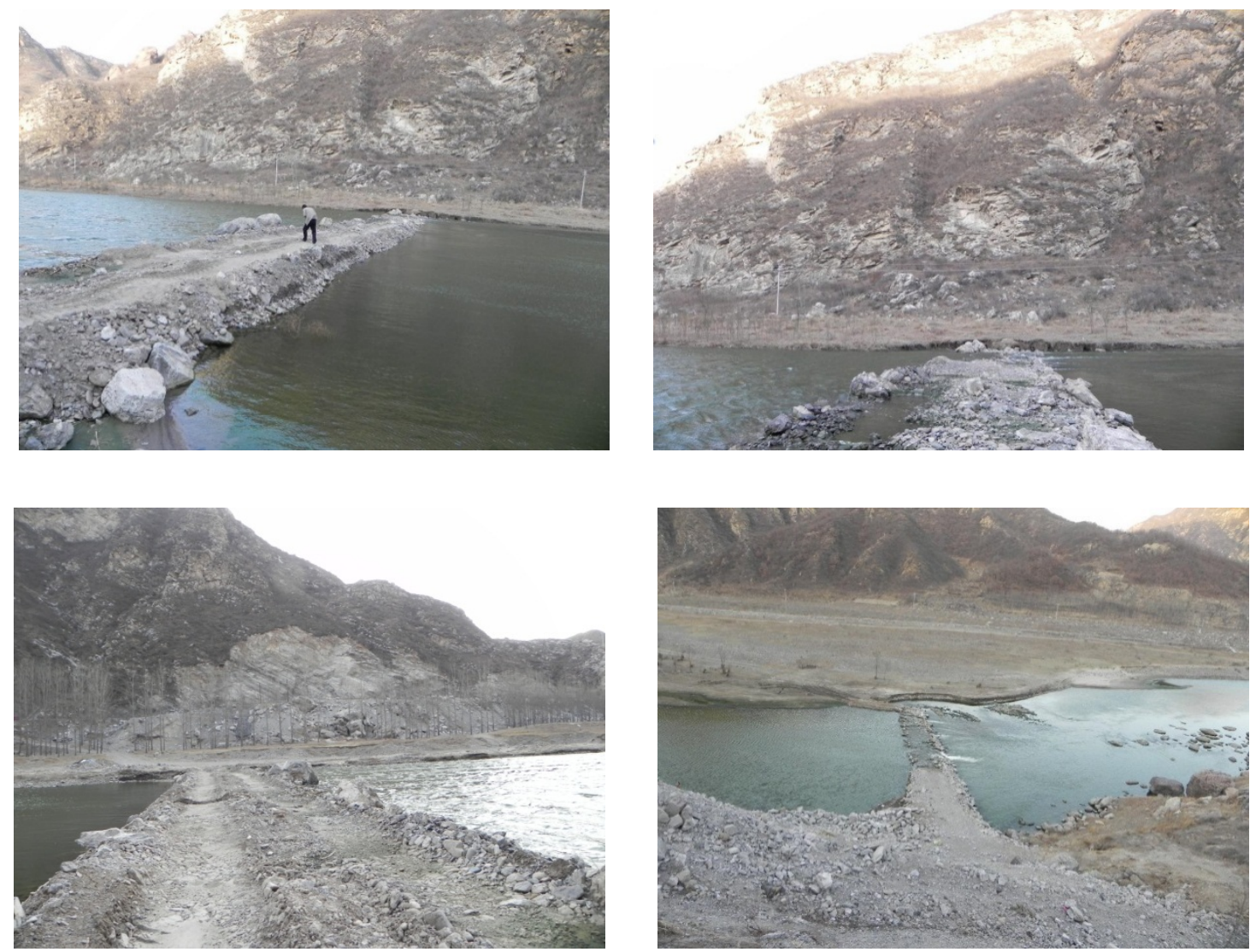

Fig. 5 The 4 sand and stone filter dams in Luan River

\section{Analysis}

\section{Monitoring}

\section{Monitoring Site.}

There are a total of 20 monitoring points around the four sand and stone filter dams, including dam 1 right upper, right lower, and dam 2 / 3 / 4 right upper, right lower, middle upper, middle lower, left upper, left lower. The monitoring points from upstream to downstream are 1-20 respectively.

\section{Monitoring Period.}

April 23-27, June 10-12, 2 times, 8-day continuous monitoring. 


\section{Monitoring Projects.}

Total nitrogen, ammonia nitrogen, total phosphorus.

\section{Effect Analysis}

From Fig. 6-Fig. 8, it can be clearly seen that the sand filter dams have a good reduction effect on the total nitrogen, ammonia nitrogen, and total phosphorus in the river.

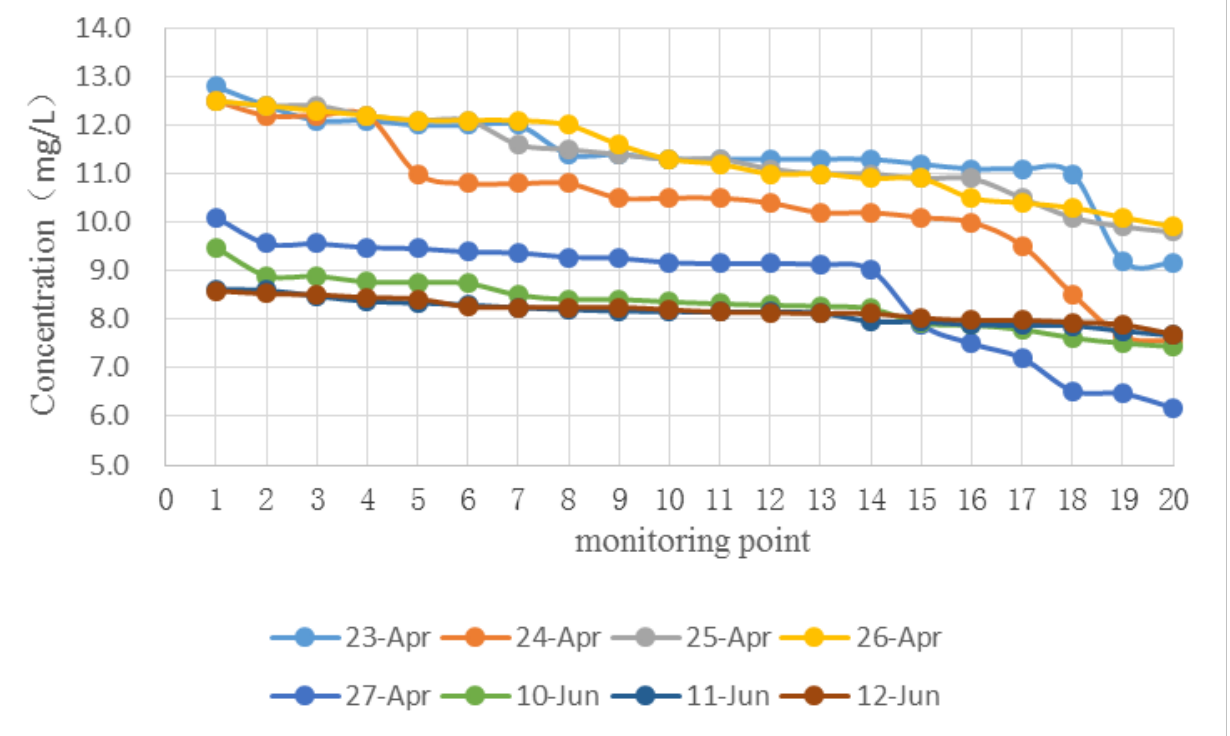

Fig. 6 The trend of total nitrogen reduction through four dams

From the above figure, it can be seen that the total nitrogen has a significant reduction effect after passing through the sand filter dam, with a maximum reduction rate of $39.52 \%$, from $12.50 \mathrm{mg} / \mathrm{L}$ to $7.56 \mathrm{mg} / \mathrm{L}$, and the lowest reduction rate is more than $10 \%$.

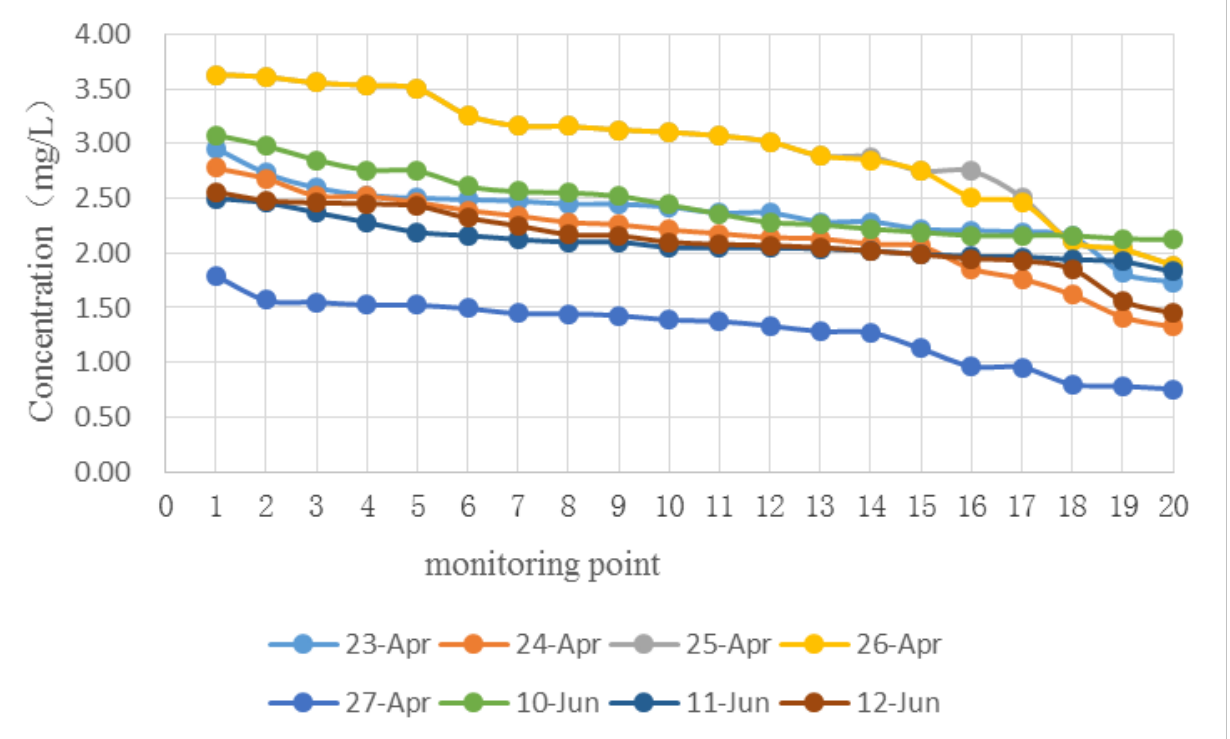

Fig. 7 The trend of ammonia nitrogen reduction through four dams

As can be seen from the above figure, the ammonia nitrogen has been reduced obviously after four dams, the highest reduction rate is $57.81 \%$, from $1.79 \mathrm{mg} / \mathrm{L}$ to $0.76 \mathrm{mg} / \mathrm{L}$, the lowest reduction rate is more than $26 \%$. 


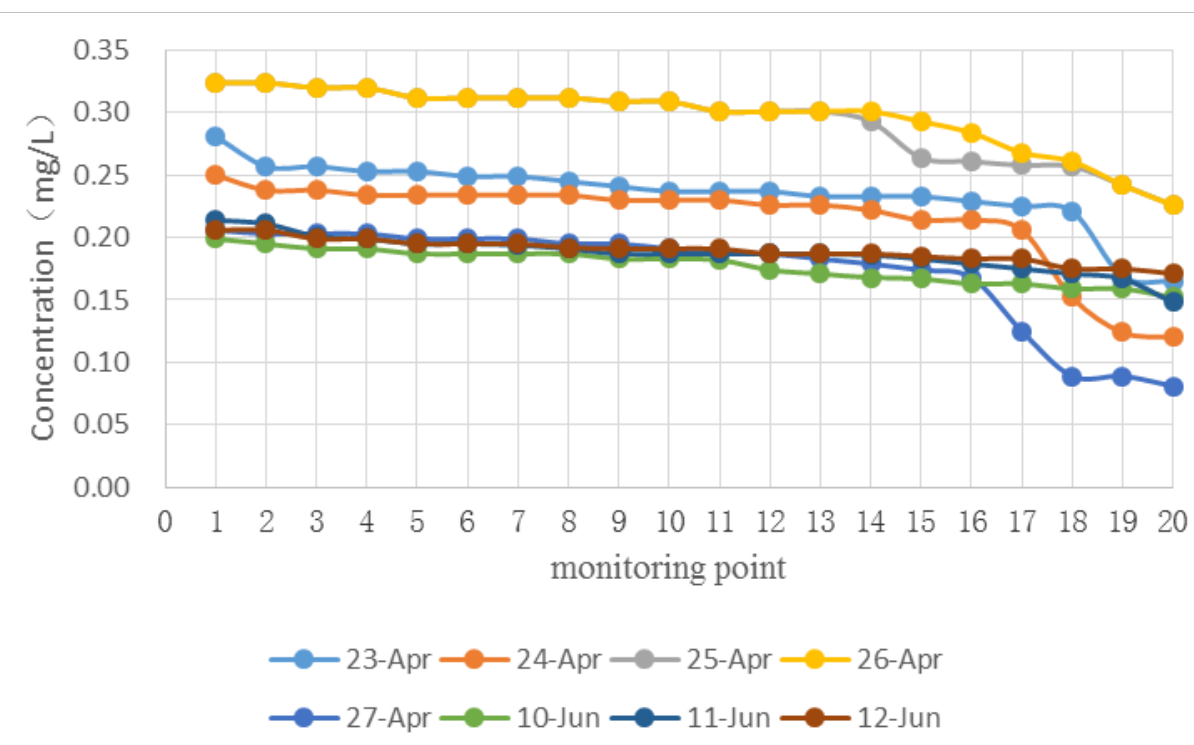

Fig. 8 The trend of total phosphorus reduction through four dams

It can be seen from the above image that the total phosphorus after four dams has obvious effect, the highest cutting rate is $60.68 \%$, from $0.206 \mathrm{mg} / \mathrm{L}$ to $0.081 \mathrm{mg} / \mathrm{L}$, the lowest reduction rate is more than $16 \%$.

\section{Discussion}

Sand and stone filter dam has the advantages of high reduction rate of nitrogen and phosphorus, and obvious effect of improving water quality. It has many advantages, such as compact structure, simple and easy operation, easy maintenance and low operating cost. However, the mechanism of purifying water quality of sand and stone filter dam needs to be further studied.

Sand and stone filter dam is only suitable for water with low flow rates. And the dam is too easy to be washed away by flood. The four sand and stone filter dams were destroyed due to flooding, the study on the service life of the dams has not yet been carried out. And further research, whether their abatement capacity will decrease with the increase of time, is also to be further studied.

\section{References}

[1] FENG Sijun, Study on water quality analysis and protection countermeasures of water source from Luan River in Tianjin, Water Resources Development Research. 9 (2014) 55-59. 